\title{
Performance Improvement of OFDM by 2-Dimensional Wavelet Transform in Flat Fading Channel
}

\author{
Salih M. Salih \\ Electrical Dept./ College of Eng. \\ University of Anbar/ Iraq \\ Tel: 009647901255958 \\ dr_salih_moh@yahoo.com
}

\author{
Mohammed A. Hussein \\ Electrical Dept./ College of Eng. \\ University of Anbar/ Iraq \\ Tel: 009647901573394 \\ elecom1978@yahoo.com
}

\author{
Abdul-Salam M. Abboud \\ Electrical Dept./ College of Eng. \\ University of Anbar/ Iraq \\ Tel: 009647807790204
}

\begin{abstract}
In this paper, a new structure was proposed by replacing the blocks of the fast Haar Wavelet Transform (WT) with a two dimensional wavelet transform at the transmitter and the receiver sides in the Orthogonal Frequency Division Multiplexing (OFDM) model. This can be done by converting a 1-Dimensional vector into a 2-dimentional matrix and process it by 2Dimentional wavelet transform (2-DWT). The proposed method was applied on the OFDM in Additive White Gaussian Noise (AWGN) and flat fading channel. It was concluded that the proposed method gives much better Bit Error Rate (BER) performance than the conventional OFDM model based on WT. The simulation results showed that the new structure outperforms the other scheme in the carried tests at the AWGN and flat fading channels.
\end{abstract}

\section{General Terms}

Algorithms, Performance, Design, Theory.

\section{Keywords}

Two-Dimensional, Wavelet Transform, OFDM, Flat Fading, AWGN.

\section{INTRODUCTION}

Orthogonal Frequency Division Multiplexing (OFDM) is a special technique of multicarrier modulation, where a single data stream is transmitted over a number of lower rate subcarriers. It is worth mentioning here that OFDM can be seen as either a modulation technique or a multiplexing technique. One of the main reasons to use OFDM is to increase the robustness against frequency selective fading and the narrowband interference. In a single carrier system, a single fade or interferer can cause the entire link to fail, but in a multicarrier system, only a small percentage of subcarriers will be affected. Error correcting coding can then be used to correct the few erroneous subcarriers. The first scheme for OFDM was proposed by Chang in 1966 [1] for dispersive fading channels. OFDM was standardized as the European Digital Audio Broadcasting (DAB) as well as Digital Video Broadcasting (DVB) scheme [2]. It constituted also a credible proposal for the recent third-generation mobile radio standard competition in Europe; also OFDM was recently selected as the HIgh PERformance Local Area Network (HIPERLAN) transmission technique as well as becoming part of IEEE 802.11 WLAN standard [3]. In the OFDM system the bandwidth is divided into high narrow sub-bands in which the mobile channel can be considered no dispersive. Since no channel equalizer is required and instead of implementing a bank of sub- channel modems they can be conveniently implemented with the aid of Fast Fourier Transform (FFT) [3]. The employment of the Discrete Fourier Transform (DFT) to replace the banks of sinusoidal generators and the demodulators that had suggested by Weinstein and Ebert [4] in 1971, which significantly reduces the implementation complexity of OFDM modems, also they conceived the guard interval to avoid the Inter-Symbol Interference (ISI) and the Inter-Carrier Interference (ICI). This proposal opened a new era for OFDM.

In 2004 Zhang, et al [5] carried out research on DFT-OFDM and Discrete Wavelet Transform-OFDM (DWT-OFDM) on different transmission scenarios. The DFT based OFDM has currently drawn most attention in the area of wireless communication. To combat ISI, and ICI, cyclic prefix is inserted between DFTOFDM symbols, and this will take up nearly 25 percent of bandwidth. To improve the bandwidth efficiency and to reduce the ISI, ICI, DWT-OFDM was proposed. Salih M., et al [6] was proposed a new model for Multicarrier-Code Division Multiple Access (MC-CDMA) based on In-Place Wavelet Transform (IPWT). The ordered Haar wavelet transform requires additional arrays at each sweep, and it assumes that the whole sample is known at the start of the algorithm, in contrast, some applications require real-time processing as the signal proceeds, which precludes any knowledge of the whole sample, and some applications involve arrays so large that they do not allow sufficient space for additional arrays at each sweep. The two problems just described, lack of time or space, have a common solution in the In-Place Fast Haar Wavelet Transform [6, 7, and 8], which differs from the ordered Haar wavelet transform algorithm only in its indexing scheme.

The Haar's wavelets are extended from One-Dimensional to Multi-Dimensional grid of data, for instance, encodings of photographs, scatter plots, or geographical measurements. Some of the logical derivations involve matrix algebra. For twodimensional wavelet, encodings can consist of matrices, indexed by rows from top to bottom, and by columns from left to right. Corresponding to the index of the rows, the first coordinate axis runs from top to bottom; similarly corresponding to the index of the columns, the second coordinate axis runs from left to right. Such an indexing scheme amounts to a rotation of the usual mathematical axes by one quarter of a turn clockwise, as shown in fig. (1) [8]. In OFDM system the input signal to the FFT or WT consist of one-dimensional vector of length $1 \times N$, where $\mathrm{N}$ is the size of FFT, in this paper, the modulated data and training vectors will be converted into two-dimensional matrix and process them as 2-dimensional matrix by WT, then it will be converted into one-dimensional vector in order to be suitable to send them as a 
serial transmission signal over the wireless channel to the receiver.

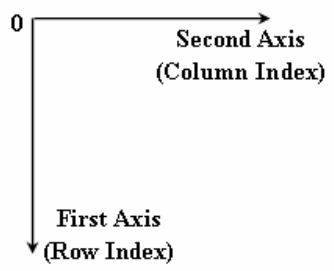

Figure 1. Axes matching indices of rows and columns.

\section{TWO-DIMENSIONAL ANALYSIS}

A one-dimensional fast Haar wavelet transform extends to a twodimensional fast Haar wavelet transform with tensor products, through alternating applications of the one-dimensional transform to each row and then to each new column.

\subsection{Two-Dimensional Approximation with Step Function}

In terms of image processing, the wavelet transform is created by repeatedly filtering the image coefficients on a row-by-row and column-by-column basis. After each transform pass (once through each row and column), the low pass coefficients of the image may be transformed again. This process can be repeated until the height or width of the area to be transformed is no longer divisible by two. Just as a simple step functions approximate functions in one dimension. Simple square-step functions approximate functions in two dimensions. Also, as a basic one-dimensional step functions has the value 1 in an interval and zero everywhere else, each basic square-step function has the value 1 in a selected square, and zero everywhere else. (For graphing purposes, their graph lies over the two dimensional square in a three-dimensional space, as in fig. (2) [8].

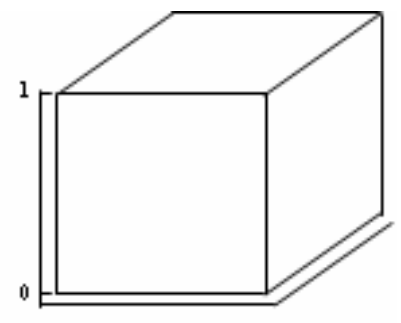

Figure 2. the simple square-step function $\Phi_{0,0}^{(0)}$

The step function $\Phi_{0,0}^{(0)}$ in fig. (2) is defined by

$\Phi_{0,0}^{(0)}(x, y)= \begin{cases}1 & \text { if } 0 \leq x<1 \text { and } 0 \leq y<1 \\ 0 & \text { otherwise. }\end{cases}$

\subsection{Tensor Products of Functions}

One of the methods to extend one-dimensional wavelets to two dimensional wavelets employs the products of basic wavelets in the first dimension with basic wavelets in the second dimension.

Definition: for each pair of functions $p$ and $q$, the tensor product of $\mathrm{p}$ and $q$ is the function denoted by $p \otimes q$ and defined by:

$$
(p \otimes q)(x, y)=p(x) \cdot q(y) .
$$

\subsection{The Basic Two-Dimensional Haar WT}

Consider a function $f$ approximated by a square step function $\bar{f}$, and encoded by a matrix [8]:

$$
\bar{f}=\left(\begin{array}{cc}
f(0,0) & f\left(0, \frac{1}{2}\right) \\
f\left(\frac{1}{2}, 0\right) & f\left(\frac{1}{2}, \frac{1}{2}\right)
\end{array}\right)=\left(\begin{array}{ll}
S_{0,0} & S_{0,1} \\
S_{1,0} & S_{1,1}
\end{array}\right)
$$

The matrix just displayed the encoded values of $f$, but for some applications, pattern of change prove more useful than the values. One method to calculate such patterns expresses the same function in terms of two-dimensional wavelets. For instance, one algorithm begins with one- dimensional wavelet transform of each row,

$$
\left(\begin{array}{ll}
S_{0,0} & S_{0,1} \\
S_{1,0} & S_{1,1}
\end{array}\right) \rightarrow\left(\begin{array}{ll}
\frac{S_{0,0}+S_{0,1}}{2} & \frac{S_{0,0}-S_{0,1}}{2} \\
\frac{S_{1,0}+S_{1,1}}{2} & \frac{S_{1,0}-S_{1,1}}{2}
\end{array}\right)
$$

and then a one-dimensional wavelet transform on each new column,

$$
\begin{aligned}
& \left(\begin{array}{cc}
\frac{S_{0,0}+S_{0,1}}{2} & \frac{S_{0,0}-S_{0,1}}{2} \\
\frac{S_{1,0}+S_{1,1}}{2} & \frac{S_{1,0}-S_{1,1}}{2} \\
\downarrow & \downarrow
\end{array}\right) \\
& \left(\begin{array}{ll}
\frac{\left(S_{0,0}+S_{0,1}\right)+\left(S_{1,0}+S_{1,1}\right)}{4} & \frac{\left(S_{0,0}-S_{0,1}\right)+\left(S_{1,0}-S_{1,1}\right)}{4} \\
\frac{\left(S_{0,0}+S_{0,1}\right)-\left(S_{1,0}+S_{1,1}\right)}{4} & \frac{\left(S_{0,0}-S_{0,1}\right)-\left(S_{1,0}-S_{1,1}\right)}{4}
\end{array}\right)
\end{aligned}
$$

In order to illustrate the steps of two-dimensional fast Haar wavelet transform, let us consider a vector of length $\mathrm{N}=16$ bit with values of $[9,7,6,2,5,3,4,4,8,2,4,0,6,0,2,2]$, the steps in the proposed method are: 
1) Convert the modulated vector of length $1 * N$ into a square matrix form with a dimension of $M \times M$, where $M \times M=1 \times N$, i.e. $M=\sqrt{N}$. In this example, the discrete values of function $\mathrm{f}$ sampled at $4 * 4=16$ values on a square grid without modulation, and approximated by the corresponding square-step function as:

$$
\bar{f}=\left(\begin{array}{llll}
9 & 7 & 6 & 2 \\
5 & 3 & 4 & 4 \\
8 & 2 & 4 & 0 \\
6 & 0 & 2 & 2
\end{array}\right)
$$

2) The first step of the two dimensional transform consist of a first one-dimensional step for each row,

$$
\left(\begin{array}{llll}
9 & 7 & 6 & 2 \\
5 & 3 & 4 & 4 \\
8 & 2 & 4 & 0 \\
6 & 0 & 2 & 2
\end{array}\right) \rightarrow\left(\begin{array}{llll}
\frac{9+7}{2} & \frac{9-7}{2} & \frac{6+2}{2} & \frac{6-2}{2} \\
\frac{5+3}{2} & \frac{5-3}{2} & \frac{4+4}{2} & \frac{4-4}{2} \\
\frac{8+2}{2} & \frac{8-2}{2} & \frac{4+0}{2} & \frac{4-0}{2} \\
\frac{6+0}{2} & \frac{6-0}{2} & \frac{2+2}{2} & \frac{2-2}{2}
\end{array}\right)=\left(\begin{array}{llll}
8 & 1 & 4 & 2 \\
4 & 1 & 4 & 0 \\
5 & 3 & 2 & 2 \\
3 & 3 & 2 & 0
\end{array}\right)
$$

3) Then, followed by a first one-dimensional step for each new column,

$$
\begin{aligned}
& \left(\begin{array}{llll}
8 & 1 & 4 & 2 \\
4 & 1 & 4 & 0 \\
5 & 3 & 2 & 2 \\
3 & 3 & 2 & 0
\end{array}\right) \\
& \left(\begin{array}{cccc}
\frac{8+4}{2} & \frac{1+1}{2} & \frac{4+4}{2} & \frac{2+0}{2} \\
\frac{8-4}{2} & \frac{1-1}{2} & \frac{4-4}{2} & \frac{2-0}{2} \\
\frac{5+3}{2} & \frac{3+3}{2} & \frac{2+2}{2} & \frac{2+0}{2} \\
\frac{5-3}{2} & \frac{3-3}{2} & \frac{2-2}{2} & \frac{2-0}{2}
\end{array}\right)=\left(\begin{array}{cccc}
6 & 1 & 4 & 1 \\
2 & 0 & 0 & 1 \\
4 & 3 & 2 & 1 \\
1 & 0 & 0 & 1
\end{array}\right)
\end{aligned}
$$

The intermediate result just obtained corresponds to one basic two dimensional wavelet transform on each of the four adjacent $2 * 2$ squares. Hence two methods become available to complete the two dimensional transform: In-place or ordered, as done here.

4) Before proceeding to the second two-dimensional step, rearrange the intermediate result just obtained to collect similar wavelets together.

$$
\left(\begin{array}{llll}
6 & 1 & 4 & 1 \\
2 & 0 & 0 & 1 \\
4 & 3 & 2 & 1 \\
1 & 0 & 0 & 1
\end{array}\right) \rightarrow\left(\begin{array}{llll}
6 & 4 & 1 & 1 \\
4 & 2 & 3 & 1 \\
2 & 0 & 0 & 1 \\
1 & 0 & 0 & 0
\end{array}\right)
$$

5) Perform a Two-dimensional wavelet transform only on the four entries in the upper left hand corner.

$$
\begin{aligned}
\left(\begin{array}{ll}
6 & 4 \\
4 & 2
\end{array}\right) \rightarrow\left(\begin{array}{cc}
\frac{6+4}{2} & \frac{6-4}{2} \\
\frac{4+2}{2} & \frac{4-2}{2}
\end{array}\right) & =\left(\begin{array}{cc}
5 & 1 \\
3 & 1
\end{array}\right) \\
\downarrow & \downarrow \\
& \left(\begin{array}{cc}
\frac{5+3}{2} & \frac{1+1}{2} \\
\frac{5-3}{2} & \frac{1-1}{2}
\end{array}\right)=\left(\begin{array}{ll}
4 & 1 \\
1 & 0
\end{array}\right)
\end{aligned}
$$

Thus emerges the completed two-dimensional wavelet transform:

$$
\left(\begin{array}{llll}
4 & 1 & 1 & 1 \\
1 & 0 & 3 & 1 \\
2 & 0 & 0 & 1 \\
1 & 0 & 0 & 0
\end{array}\right)
$$

\section{SIMULATION MODEL}

In this section, the proposed OFDM transceiver based on twodimensional Haar wavelet transform will be described, and its performance will be discussed. Fig. (3) [3, 9], shows the traditional OFDM based on wavelet transform and the proposed model is shown in fig. (4). It can be seen that the Inverse Wavelet Transform (IWT) and the Wavelet Transform (WT) blocks in fig. (3) are replaced by a 2-Dimensional Inverse Wavelet Transform at the transmitter and 2-Dimensional Wavelet Transform at the receiver side as in fig. (4).

From fig. (3), the input data stream is first mapped into Qudarature Amplitude Modulation (QAM) according to the QAM constellation mapping of m-points, and then the output complex signal is converted from serial to parallel into $\mathrm{N}$-points wavelet transform to generate the OFDM symbol. The output data from the WT block is now converted from parallel to serial. The data are sent to the receiver over the channel after being converted to a frame structure (serial data stream). The frame structure consists of modulated data and the pilot signal which is used for channel estimation and compensation. The channel consists of a multipath fading (flat fading channel or frequency selective fading channel) with AWGN, at the receiver the inverse operation is employed. A serial to parallel conversion is done to the received signal. A wavelet transform with $\mathrm{N}$ points is used to convert the signal at the receiver as an inverse operation of the Inverse WT at the transmitter side. Then the effective channel is compensated by using the pilot signals. The signal de-mapper is used to recover the transmitted signal. The training sequence will be used to estimate the channel frequency response as follows $[6,10]$ :

$$
H(k)=\frac{\text { Received Training Sample }(k)}{\text { Transmitted Training Sample }(k)}
$$


The channel frequency response will be used to compensate the channel effects on the data, and the estimated data can be found using the following equation:

$$
\text { Estmated. data }=H^{-1} \text { estimate }(k) * \text { Re ceived.data }(k)
$$

For the proposed model shown in fig. (4), the output data and training are converted from one dimensional vector into twodimensional matrix (square matrix), then both of data and training signals are processed by a 2-Dimensional Inverse Wavelet Transform (2-DIWT). The data and training signals are converted from 2-dimensional matrix into a one vector of length $\mathrm{N}$ for each of them. At the receiver the inverse operation will be applied on both of data and training signals in order to recover the transmitted signal.

Hint: in this simulation the data and training signals have a size of $\mathrm{N}=64$ points, so the matrix size will be of size $=8 * 8$. if we would like to make a simulation of size $\mathrm{N}=32$ then it can be use two matrices of size $4 * 4$ for each one to get two square matrices that will be compatible with a vector of length $\mathrm{N}=32$ point.

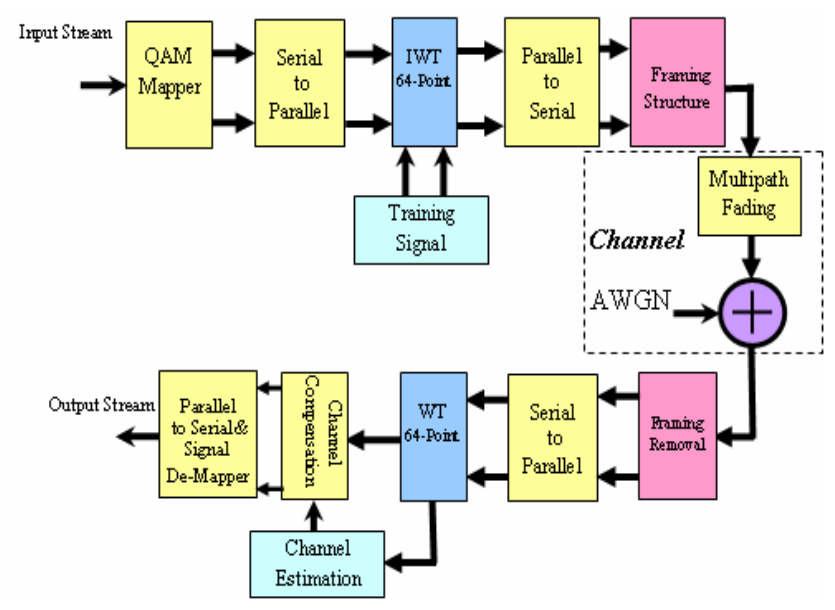

Figure 3. Traditional OFDM model based on WT.

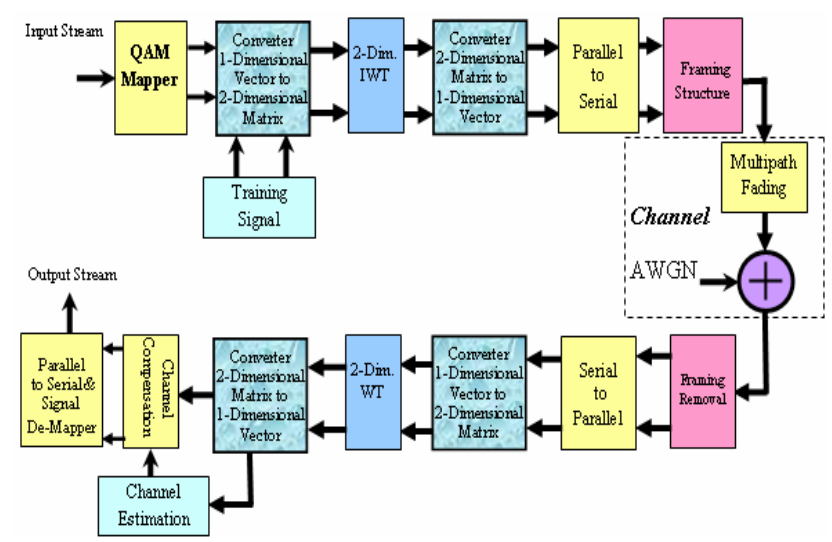

Figure 4. A proposed OFDM model based on 2-Dimensional wavelet transforms.

\section{SIMULATION RESULTS}

In this section, the combination of the conventional OFDM with the proposed OFDM based on 2-DWT will be studied, in this research a 64 bit of data and training for each one will be used. A simulation of the two systems has been made using MATLAB 7. The BER performance of the two systems will also be studied in two types of models of channels which are AWGN, and AWGN+flat fading channel, with a bit rate of 5 Mbps and 64 subcarriers are used in this simulation. The simulation parameters are given in table 1.

Table 1. Simulation Parameters

\begin{tabular}{|c|c|}
\hline $\begin{array}{l}\text { Modulation Type and the } \\
\text { number of constellation points }\end{array}$ & $\begin{array}{c}\text { QAM, m=8 Point and } \\
\text { m=64 Point }\end{array}$ \\
\hline Number of sub-carriers & 64 \\
\hline Number of WT points & 64 \\
\hline Doppler Frequency $\left(f_{d}\right)$ & $5 \mathrm{~Hz}, 200 \mathrm{~Hz}$ \\
\hline Data rate & 5Mbps \\
\hline \multirow[b]{2}{*}{ Channel model } & AWGN \\
\hline & Flat fading+AWGN \\
\hline
\end{tabular}

\subsection{Performance of the Proposed System in AWGN Channel}

The channel here is modeled as an Additive White Gaussian Noise for wide range of SNR from $0 \mathrm{~dB}$ to $40 \mathrm{~dB}$. From Fig. (5), it is found that the proposed OFDM system based on 2-DWT worked with $\mathrm{SNR}=17 \mathrm{~dB}$ at $\mathrm{BER}=10^{-4}$ when the number of constellation points $m=8$, while in the traditional OFDM the SNR at $\mathrm{BER}=10^{-4}$ is $23 \mathrm{~dB}$, which means a gain of $6 \mathrm{~dB}$ is obtained by the proposed model over the traditional one. As the number of constellation points increase to 64 point, then the BER will also increases for both of the traditional and the proposed model as shown in the dotted lines. The obtained gain is $6 \mathrm{~dB}$ that appears from the proposed system over the conventional one at $\mathrm{BER}=10^{-4}$. In all SNR range values, the proposed model has better performance than the conventional system.

\subsection{Performance of the Proposed System in Flat Fading Channel}

The simulation results for both systems in flat fading channel are shown in fig.(6) and fig. (7). Two values of the Doppler frequencies (fd) are considered in this simulation, these are $\mathrm{fd}=5$ $\mathrm{Hz}$ and $200 \mathrm{~Hz}$ respectively. From fig. (6), it can be seen that the proposed OFDM still performs better than the traditional OFDM in all range of $\mathrm{SNR}$, where it approaches the $\mathrm{BER}=10^{-4}$ at $\mathrm{SNR}=22 \mathrm{~dB}$ and $28 \mathrm{~dB}$ when $\mathrm{m}$ has increased from 8 to 64 point. The conventional model based on WT has BER $=25 \mathrm{~dB}$ at $\mathrm{m}=8$ and $37 \mathrm{~dB}$ at $\mathrm{m}=64$ point. The obtained gains from the proposed model at these cases are $3 \mathrm{~dB}$ and $9 \mathrm{~dB}$ at $\mathrm{BER}=10^{-4}$.

The BER increases as the Doppler frequency increases for both models as shown in fig. (7). The SNR required maintaining a BER at $10^{-4}$ increased by about $15 \mathrm{~dB}$ from $22 \mathrm{~dB}$ in fig. (6) to $37 \mathrm{~dB}$ in fig. (7) as the Doppler frequency increased from 5 to 200 $\mathrm{Hz}$ in the proposed model. The BER is more than $10^{-4}$ in the 
simulated SNR range at $\mathrm{m}=64$ point. The proposed model outperforms the traditional model at $\mathrm{m}=8$ and 64 point.

Finally, the proposed and traditional model have unacceptable performance in selective fading channel due to the effect of multipath on the transmitted symbols, this weakness point of their performances can simply be solved by combining OFDM with antenna arrays at the transmitter and receiver to increase the diversity gain and/or to enhance the system capacity on timevariant and frequency-selective channels, resulting in a MultipleInput Multiple-Output (MIMO) configuration [11], this method can enhance the system performance in selective fading channel by using MIMO-OFDM structure with the proposed algorithm. Also the processing time can be reducing it by using a TwoDimensional WT based on In-Place WT instead of the ordered WT that was used in this simulation $[6,8]$. The In-Place WT requires a minimum memory size for each sweep with a fast processing time.

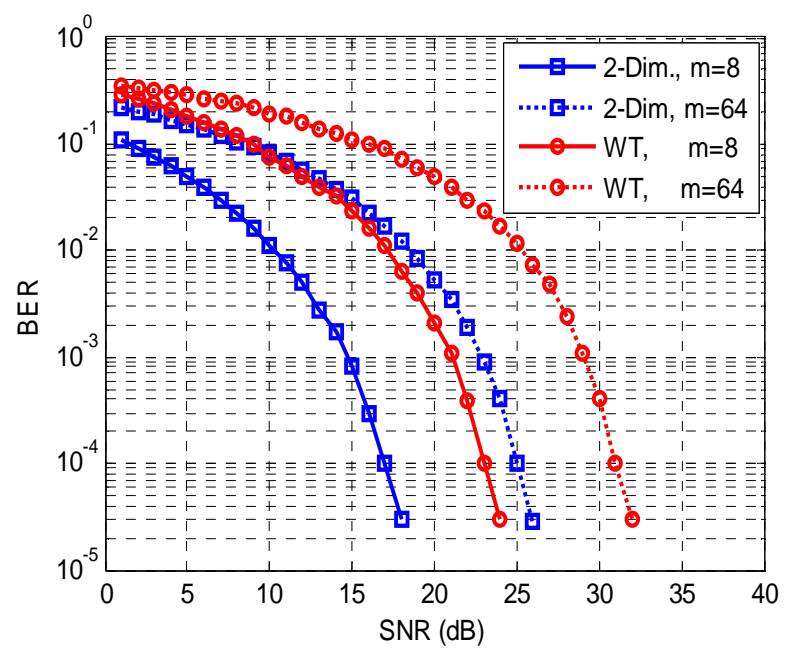

Figure 5. Performance of the proposed and traditional model in AWGN channel.

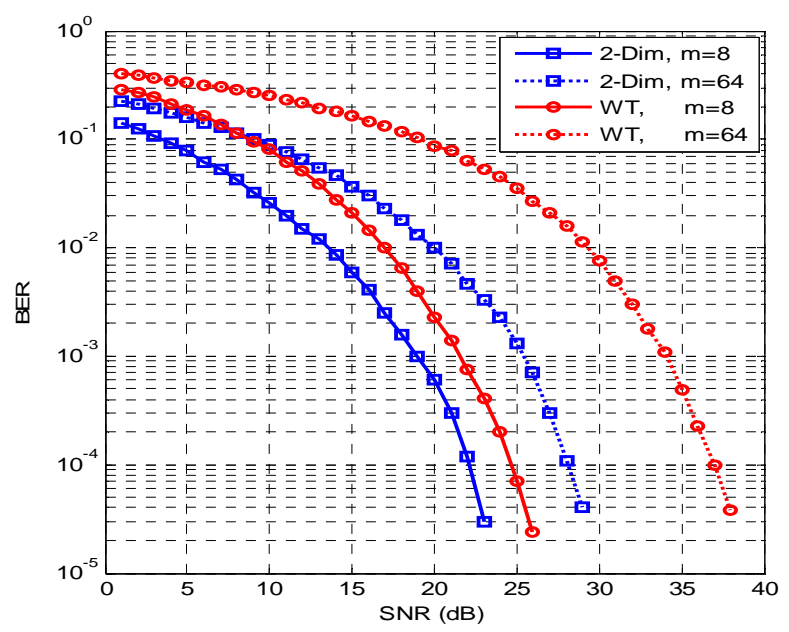

Figure 6. Performance of the proposed and traditional model in flat fading channel $\left(f_{d}=5 \mathrm{~Hz}, \mathrm{~m}=8\right.$, and 64 point $)$.

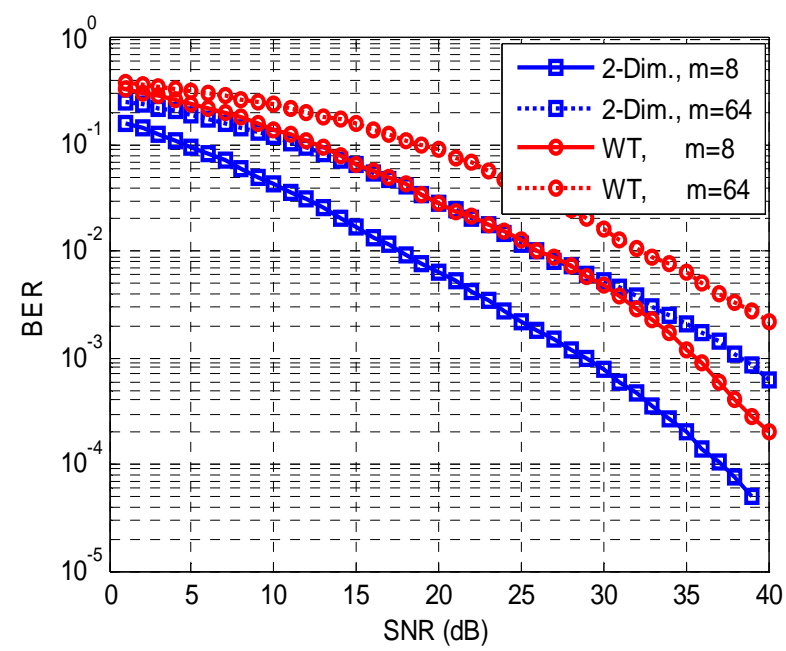

Figure 7. Performance of the proposed and traditional model in flat fading channel $\left(f_{d}=200 \mathrm{~Hz}, m=8\right.$, and 64 point $)$.

\section{CONCLUSIONS}

The simulation of the proposed and traditional OFDM systems has been investigated. It has been shown that the new algorithm based on 2-dimensional wavelet transform is widely active to work under the AWGN and flat fading channel characteristics. A gain of $6 \mathrm{~dB}$ was obtained at the AWGN channel. The obtained gains from the proposed model in flat fading channel are $3 \mathrm{~dB}$ and $9 \mathrm{~dB}$ at $\mathrm{BER}=10^{-4}$ as the number of constellation points increased from $m=8$ to $m=64$ point. The BER increases as the Doppler frequency increases for both models, at this case the proposed model has better performance than the traditional model based on the ordered Haar WT. Therefore this structure can be considered as an alternative to the conventional OFDM system. It can be concluded from the obtained results, that the SNR measure can be successfully increased by using the proposed 2-dimensional designed method within a desired parameters.

\section{REFERENCES}

[1] R. W Chang., 1966. Synthesis of Band-Limited Orthogonal Signals for Multichannel Data Transmission. Bell System Technical Journal, vol. 46, (December 1966) pp. 1775-1796.

[2] T. De. Couasnon, R Monnier, and J. B. Rault, 1994. OFDM for Digital TV Broadcasting. Journal of Signal Processing, vol. 39, (1994). pp. 1-32.

[3] L.Hanzo, M. Munster, B. J., Chol, and T. Keller, 2003. OFDM and MC-CDMA for Broadband Multi-user Communications, WLANs and Broadcasting. John Wiley \& Sons, 2003.

[4] S.B. Weinstein and P. M. Ebert, 1971. Data Transmission by Frequency Division Multiplexing Using the Discrete Fourier Transform. IEEE Transaction on Communication. Technology, vol. com-19, (October 1971). pp. 628-634. 
[5] H. Zang, Dongfeng Yuan, Mingyan Jiang and. Dalei Wu, 2004. Research of DFT-OFDM and DWT-OFDM on Different Transmission Scenarios," Proceedings of the $2^{\text {nd }}$ International Conference on Information Technology for Application (ICITA).

[6] Salih M. Salih, N. Uzunoglou, Laith Awda, and Laith A. ElAnzy, 2007. A Proposed Model for MC-CDMA Based on In-Place Wavelet Transform. Third International Mobile Multimedia Communications Conference (MobiMedia 2007 conference), 27-29 August 2007, Nafpaktos, Greece.

[7] L. Howard, O. Raymond. and Jr. Wells, 1998. Wavelet Analysis. ISBN 0-387-98383-x. Springer- Verlag New York.

[8] Nievergelt Yves, 1999. Wavelets Made Easy. Birkhauser, Boston Inc.

[9] Shinsuke Hara, and Prasad R., 2003. Multicarrier Techniques for 4G Mobile Communications. Artech House, Boston. London.

[10] Saad Nihad Abdul-Majeed, 2006 A Multiwavelet Based MCCDMA System. Ph.D. thesis, College of Engineering, Baghdad University.

[11] Gordon L. Stuber, John R. Barry, Steve W. Mclaughlin, Ye. Li., Mary Ann Ingram, and Thomas G. Pratt, 2004. Broadband MIMO-OFDM Wireless Communications," Proceedings of the IEEE, vol. 92, no. 2, (February, 2004). 